\title{
STUDIA ROMANICA POSNANIENSIA
}

\begin{tabular}{lll}
\hline UAM & Vol. 36 & Pozná́ 2009
\end{tabular}

\section{AGNIESZKA KALISKA}

Université Adam Mickiewicz, Poznań

\section{LES PRÉDICATS CAUSATIFS MORPHOLOGIQUES EN FRANÇAIS ET EN POLONAIS ${ }^{1}$}

\author{
JE DÉDIE CET ARTICLE À M. JÓZEF SYPNICKI
}

A bstract. Kaliska Agnieszka, Les prédicats causatifs morphologiques en français et en polonais [The morphological causative predicates in French and Polish]. Studia Romanica Posnaniensia, Adam Mickiewicz University Press, Poznań, vol. XXXVI: 2009, pp. 89-96. ISBN 978-83-232-2035-0. ISSN 0137-2475.

This paper focuses on a group of French and Polish morphological causative constructions in which the cause and effect elements are contiguous. One of the main claims pursued here is that their semantic deep structure is parallel to the one of the respective analytical causative constructions. On the other hand, Polish prefixes are more problematic because of their capacity to express the causation and the perfectibility by the same form. Some linguists claim that there are no causative prefixes in Polish but only flexional circumfixes with the capacity to express the causation. The paper also tackles the problem of definability of causation. Sometimes vastly different ideas are subsumed under this label.

\section{INTRODUCTION}

Le but du présent article est de comparer la forme et les traits syntaxicosémantiques des prédicats causatifs morphologiques en français et en polonais, c'est-à-dire des expressions prédicatives verbales dotées de morphèmes spécialisés pour exprimer la causativité ${ }^{2}$.

Notre étude s'inscrit dans le domaine de la morphologie constructionnelle où l'analyse du sens du mot se fait par la décomposition de celui-ci en formants,

'Le prèsent article a été écrit à la base d'une communication faite pendant le Dixième séminaire international d'études doctorales, intitulè «Forme et sens », qui s'était tenu à Podebrady en février 2006.

${ }^{2}$ Sont exclus de la présente analyse les dérivés en -oyer, suffixe causatif typique pour le français populaire (Nyrop, 1913: 211, Herslund, 1991: 136), les verbes non agentifs et les verbes pronominaux (ou posifixaux en polonais selon l'interprétation de Sękowska (1999: 188). 
«cellules signifiantes en travail» (Molho, 1982: 50). La segmentation des unités lexicales en éléments plus simples démontre quels sont les rôles que les morphèmes successifs assument ainsi que les règles qui président à leur combinaison. Dans cet article, nous voulons présenter quelques régularités et idiosyncrasies dérivationnelles qui nous ont frappés lors de l'analyse comparative des prédicats causatifs en français et en polonais.

\section{DÉINITION DE LA CAUSATIVITÉ}

La délimitation d'une classe de prédicats causatifs et leur classification n'est pas facile parce qu'elle dépend dans une large mesure de la définition de la causativité $e^{3}$. Si l'on définit la causativité comme phénomène linguistique consistant à exprimer qu'une action $A$ produit ou occasionne un effet $B^{4}$, il s'avère que la plupart des prédicats d'une langue sont causatifs parce que les actions dénotées ont toujours certaines conséquences, voulues ou involontaires.

Dans la linguistique, le terme de causativité est réservé à des expressions dont le sens lexical implique une relation causale, c'est-à-dire une relation cause-conséquence entre deux procès successifs.

\subsection{TYPES DE PRÉDICATS CAUSATIFS}

L'expression de la causativité met en jeu le lexique d'une part, l'emploi de certaines structures morphosyntaxiques d'autre part (Creissels, 1995: 286-287). Ainsi, nous avons distingué deux types de prédicats causatifs, à savoir: les prédicats causatifs réels (pol. kauzatywa realno-znaczeniowe) et les prédicats causatifs structurels (pol. kauzatywa strukturalno-znaczeniowe).

\subsubsection{CAUSATIFS RÉELS}

La causativité des prédicats causatifs réels est inscrite dans la structure sémantique profonde des lexèmes. Leur structure morphosyntaxique ne contient aucun indicateur spécifique de causativité, p. ex. : tuer 'faire mourir', enseigner 'faire savoir', nourrir 'faire manger', ainsi que les verbes déadjectivaux comme courtauder 'rendre courtaud', et les verbes dénominaux comme médailler 'décorer d'une médaille' (Jespersen, 1967: 409).

Ce groupe contient une quantité de cas discutables. Paraphrasables en 'rendre Adj' ou 'faire - Vinf', la majorité de ces verbes ne sont causatifs que dans certains

\footnotetext{
${ }^{3}$ L'on dit aussi causation (Karolak, 1991) et causalité (Creissels, 1995).

${ }^{4}$ Voir : la définition du Causativum dans Encyklopedia językoznawstwa ogólnego (1999).
} 
contextes, p.ex. : approfondir 'analyser profondément (en parlant de la question)' mais 'rendre (plus) profond (en parlant du canal)'. Il s'agit donc de deux emplois différents dont l'un est plus explicitement causatif que l'autre. De plus, les analyses approfondies ont démontré les divergences sémantiques entre deux expressions prédicatives supposées équivalentes par les sémanticiens générativistes : un prédicat causatif synthétique et sa paraphrase lexicale étant en même temps une variante analytique du prédicat n'ont pas forcément la même structure sous-jacente, p.ex. : tuer et causer la mort (Ruwet, 1972).

\subsubsection{CAUSATIFS STRUCTURELS}

Dans les causatifs structurels, par contre, l'expression de la causativité se fait de façon explicite grâce aux indicateurs morphologiques et syntaxiques de causativité comme :

- verbes auxiliaires, p.ex. : faire - Vinf ${ }^{5}$, rendre - Adj, etc. ;

- locutions conjonctives, p.ex. : de telle manière que, de telle façon que, etc.;

- radicaux, p.ex. : caus-er, déclench-er etc. (en pol. powod-ować, wywot-ać etc.), tous étant synonymes et, en même temps, variantes du prédicat [CAUSE-] ;

- affixes causatifs, p.ex.:-iser, -fier, -oyer dont la structure comporte un élément catégoriseur, le morphème flexionnel -er.

\section{PRÉDICATS VERBAUX CAUSATIFS SUFFIXÉS EN FRANÇAIS}

Il existe en français au moins deux suffixes à valeur causative, à savoir :-fier, -iser; les deux d'origine latine :-fier <-ficare, -iser <-izare (Huot, 2001: 80). Deux segments du dérivé, suffixe et radical, sont indicateurs formels des deux concepts fondamentaux de la relation causale, respectivement: cause et conséquence. Comparons :

$x$ fertiliser y [x FAIT QCH à y PAR CONSÉQUENT y EST FERTILE]

$x$ fortifier y [ $x$ FAIT QCH à y PAR CONSÉQUENT y EST FORT]

La forme synthétique du prédicat se laisse analyser en deux propositions tout comme la construction analytique :

Pierre a causé l'incendie. [pol. Piotr spowodowat pożar]

'Pierre a fait quelque chose [CAUSE]

de telle façon que l'incendie s'est déclarée [CONSÉQENCE]'

\footnotetext{
${ }^{5}$ Voir sur ce sujet Creissels (1995).
} 
Le second argument du prédicat causer a un caractère propositionnel ${ }^{6}$ et il est définissable en termes de [+CONSÉQUENCE]. La forme non propositionnelle de l'argument est une réduction superficielle du contenu propositionnel. Le même argument propositionnel, autrement dit prédicat du second ordre, est inscrit dans le radical du lexème dérivé.

\section{fertiliser = causer la fertilité / rendre fertile}

Ceci est encore mieux visible dans les verbes en -fier dont l'étymologie remonte au lat. ficere, c'est-à-dire faire. Ainsi, le suffixe -fier assume-t-il la même fonction que l'auxiliaire faire dans des constructions factitives du type faire Vinf. D'où l'idée qu'il est question de deux variantes formelles d'un même prédicat ${ }^{7}$, en l'occurrence [FERTIL-]. Comme il s'agit des variantes, la forme morphosyntaxique d'une expression causative n'influe ni sur la valence ni sur la structuration interne des arguments. Ainsi :

$$
\text { fort-i-fier (faire devenir fort }) \rightarrow \mathrm{P}(\mathrm{x}, \mathrm{y}) \text { [faire devenir (plus) fort }(\mathrm{x}, \mathrm{y}) \text { - être fort (y)] }
$$

L'interprétation causative des affixes est possible lorsqu'ils sont vitaux et productifs. Les -fier et -iser contribuent sans cesse à l'enrichissement du lexique ${ }^{8}$ : à partir du XIV siècle pour exemplifier et tyranniser jusqu'aux temps modernes pour nazifier et de nombreux hapax : gidiser, pindariser, lundiser, tantaliser (TLF).

\section{PRÉDICATS VERBAUX CAUSATIFS D'ORIGINE ÉTRANGÈRE EN POLONAIS}

Dans le système dérivationnel du polonais, l'existence des suffixes à fonction causative est discutable. Nombreux sont les verbes qui expriment la causativité et qui présentent des similitudes au niveau formel, p.ex. : amerykan-izować, palatalizować, aromat-yzować, steryl-izować ou kody-fikować, egzempli-fikować, mistyfikować, personi-fikować. La structure morphologique des verbes cités comporte

${ }^{6}$ «L'antécédent a un caractère propositionnel tout comme le conséquent: il s'est passé $\mathbf{P}$ qui a causé $\mathrm{Q}[\ldots .$.$] Le premier argument propositionnel de la relation causale est représenté en surface par$ l'un de ses arguments objets intérieurs. Le deuxième [...] a une représentation explicite. » (Karolak, 1991: 156).

${ }^{7}$ Dans la morphologie syntaxique de J.M. Rozwadowski, l'affixe exprime le genus proximum de l'action dénotée, le radical les differentia specifica de celle-ci. Il s'ensuit que les dérivés en -iser et -fier sont toutes variantes formelles du prédicat [CAUSE-]. Dans notre perspective, l'unité du sens qui permet de reconnaittre dans une expression prédicative donnée la forme d'un prédicat, êtant par sa nature abstrait, est situéc dans le radical du mot (voir aussi: Karolak, 1984).

${ }^{8}$ Moindre est la vitalité du suffixe (p.ex. : -oyer dans des dérivés nettoyer (rendre net) et foudroyer (frapper d'une foudre), moindre est la productivité - beaucoup de formations en -oyer cèdent la place à leurs doublets d'origine savante, p.ex. : plaidoyer et plaider (TLF). 
soit l'élément -fikować soit l'élément -izować (variante morphonologique -yzować). Pourtant, puisqu'il n'est pas question de mots d'origine polonaise, les grammairiens classent lesdits morphèmes parmi les éléments dits internationaux (pol. internacjonalizmy) et les mots qui les comportent parmi les mots dits adaptés (pol. wyrazy adaptowane) (Jadacka, 2005: 119 ; Nagórko, 1998: 172). Dans la plupart des cas, ce sont les mots d'origine latine dont plusieurs langues européennes contiennent des traces, p.ex. : fr. palatal-iser vs pol. palatal-izować vs ang. palatal-ize et fr. codifier vs pol. kody-fikować vs ang. codi-fy ${ }^{9}$. La frontière entre l'adaptation et l'affixation étant floue, les éléments empruntés peuvent vite devenir productifs. Ainsi, le verbe komputeryzować 'équiper d'ordinateurs' est une formation purement polonaise ayant pour base dérivationnelle le mot d'origine anglaise : komputeryzować < komputer < computer.

\section{PRÉDICATS VERBAUX CAUSATIFS PRÉFIXÉS EN POLONAIS}

A côté des éléments suffixaux (ou suffixoïdes), il existe dans la langue polonaise toute une suite de préfixes dont la fonction est causative. A savoir : $u-$, $z a-, o-, w y-$, roz- qui, en cooccurrant avec des morphèmes flexionnels -ić, -ać, contribuent à la formation des verbes causatifs. Ce type de dérivation est appelée dérivation préfixo-paradigmatique (pol. derywacja prefiksalno-paradygmatyczna) ou dérivation préfixo-suffixale (pol. derywacja prefiksalno-sufiksalna) (Jadacka, 2005: 119 ; Sękowska, 1992: 172). Toutefois, si la causativité est inscrite dans le sens du mot même ou s'il y a déjà un autre morphème causatif dans la structure du mot, le préfixe qui survient assume de plus la fonction perfective. Comparons la structure des verbes użyźnićlać (fr. fertiliser), (u)tworzyć (fr. créer) et upiększyćlać (fr. embellir), (u)fortyfikować (fr. fortifier) :

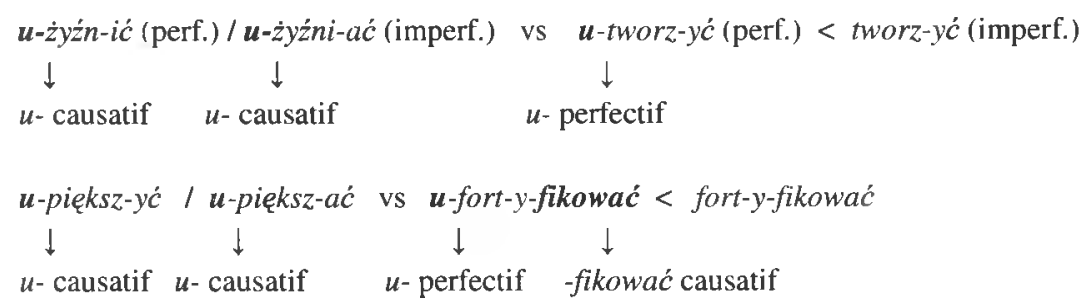

ou encore: o-chtodz-ić (perf.) et o-chładzać (imperf.) au sens de 'refroidir' comportant un $o$ - causatif qui peut pourtant être considéré également comme un morhème perfectif par rapport à l'unité chtodzić (imperf.) - tous les trois verbes ont

${ }^{9}$ Les exemples sont multiples. Pourtant, certains ne trouvent pas leurs équivalents structuraux en polonais. Le verbe franc-yzować n'existe pas, parce qu'un autre exprime dejă le sens de 'revêtir d'un caractère français', à savoir : franc-urzyć. 
pour équivalent en français le verbe refoidir. Comparons-le encore à o-bandażow$a c ́$ (perf.) avec un $o$ - perfectif, dérivé du verbe bandażow-ać (imperf.), les deux ayant pour équivalent en français le verbe bander. Là où la perfectivité est exprimable à l'aide d'un suffixe verbal -ić (éventuellement -yć) (p.ex.: ochtodzić, użyźnić, upiekszyć), le préfixe assume une fonction causative et c'est de cette façon que la définissent les dictionnaires. La question se pose : s'agit-il d'une et même forme préfixale à laquelle correspondent plusieurs structures et fonctions ? C'est un des problèmes que la morphologie constructionnelle cherche à résoudre (Mok, 1983: 70 ; Temple, 1996: 122).

\subsection{Y A-T-IL DES CIRCUMFIXES CAUSATIFS EN POLONAIS ?}

Pour remédier aux idiosyncrasies présentées ci-dessus ou « caprices » (Corbin, 1991: 10) du système dérivationnel, certains proposent de parler le cas échéant de la dérivation circumfixale ${ }^{10}$ qui se distinguerait de la dérivation préfixale par le fait qu'elle n'a jamais les verbes pour bases dérivationnelles mais les adjectifs (Nagórko, 1998: 179). Comme le polonais est une langue aspectuelle, le circumfixe aurait deux formes : l'une causative imperfectivisante $u$ - $-a c$ et l'autre causative perfectivisante $u$ - -ić (var. : $u$ - -yć). Comparons :

fr. fertiliser, en pol, $u$-żyźn-ić (v. perf.) / u-żyźni-ać (v. imperf.) < żyzny (adj. fertile)

fr. embellir, en pol. u-piększ-yć (v. perf.) / u-piększ-ać (v. imperf.) < piękny (adj. beau)

contrairement à :

fr. créer, en pol. u-tworzyć (v. perf.) < tworzyć (v. imperf.)

Effectivement, il n'existe en polonais ni le verbe *izyźnić, ni *piększyć. Pourtant, pour parler d'un circumfixe causatif, il semble que les deux segments, initial et final, doivent contribuer à l'interprétation causative du verbe (Song, 1996). Comme l'élément final -ićl-ać est l'indicateur d'une forme infinitivale du verbe polonais, perfective ou imperfective, il s'agit plutôt de la dérivation préfixo-paradigmatique.

\section{2. POLYFONCTIONNALITÉ DES PRÉFIXES VERBAUX EN POLONAIS}

Outre les problèmes cités, la difficulté dans l'interprétation des préfixes découle aussi de leur origine prépositionnelle. Grammaticalisés, les préfixes sont chargés de diverses nuances sémantiques. Ainsi le préfixe $o(b)$ - défini comme causatif (et perfectivisant) est-il porteur d'un sens paraphrasable en 'rond' ou 'alentour'. Ce sens, quoique difficilement perceptible en synchronie, fait partie du

${ }^{10}$ Les circumfixes sont des morphemes discontinus dont une partie précéde le radical du mot et l'autre le suit. 
sémème des verbes qui le comportent (p.ex. : obandażować 'bander'). Souvent, un prédicat a plusieurs variantes préfixales qui véhiculent diverses nuances sémantiques d'une action, p.ex. : szyć 'coudre' vs zszyć, zaszyć, obszyć, wszyć, wyszyć, doszyć, przeszyć, naszyć, poszyć, przyszyć, variantes traduisibles en français uniquement par des paraphrases mettant en cause divers aspects de l'action de coudre. Il est discutable laquelle des fonctions citées est primaire. Une des interprétations possibles est que le sens causatif du prédicat préfixé n'est pas l'affaire du préfixe lui-même mais du lexème entier. Le point de vue pareil s'impose dans l'analyse de la relation forme-sens des verbes préfixés en français, comme : affaiblir 'rendre (plus) faible', intimider 'rendre (plus) timide', endurer 'rendre (plus) dur', refroidir 'rendre (plus) froid'.

\section{CONCLUSION}

L'identification et la classification des unités fonctionnelles, affixes et radicaux, est problématique. Souvent, le sens du mot n'est pas prédictible à partir de sa structure interne. À un affixe correspondent souvent plusieurs structures et plusieurs sens : un même sens peut être exprimé par plusieurs procédés morphologiques (Mok, 1983: 70, Temple, 1996: 122, Nagórko, 1998: 177). Selon certains, les cas résiduels, i.e. les mots qui résistent aux règles et apparaissent plutôt comme exceptions, ne nient pas le caractère systémique de la dérivation (Huot, 2001: 16). Plus la vitalité de l'affixe est grande, plus la corrélation entre forme et sens du prédicat est visible.

\section{BIBLIOGRAPHIE}

Corbin D. (1990), « Homonymie structurelle et définition des mots construits », Langue et Langage : Définition, Paris : Larousse, p. 175-192.

Corbin D. (1991), «La formation des mots: structures et interpretations », Lexique, $\mathrm{n}^{\circ} 10$, Villeneuve d'Ascq: Presses Universitaires du Septentrion, p. 7-30.

Creissels D. (1995), Eléments de syntaxe générale, Paris : PUF.

Herslund M. (1991), "Construction causative et verbes causatifs", Analyse et synthése dans les langues romanes et slaves, dir. : H. Stammerjohann, Tübingen: Gunter Narr, p. 135-147.

Huot H. (2001), Morphologie. Forme et sens des mots du français, Paris : Armand Colin.

Jadacka H. (2005), Kultura jęzka polskiego. Fleksja, slowotwórstwo, składnia. Warszawa : PWN.

Jespersen O. (1967), La philosophie de la grammaire, Paris : Gallimard.

Karolak S. (1984), «Składnia wyrażeń predykatywnych », in : Sktadnia, dir. Z. Topolińska, Warszawa : PWN.

Karolak S. (1991), «Sur la structure sémantique des constructions factitives synthẻtiques et analytiques », in Analyse et synthese dans les langues romanes et slaves, dir. : H. Stammerjohann, Tübingen : Gunter Narr, p. 149-161. 
Leeman-Bouix D. (1994), Grammaire du verbe français: des formes aux sens, Paris : Nathan.

Lehmann A., Martin-Berthet F. (2000), Introduction à la lexicologie: sèmantique et morphologie, Liège: Nathan.

Mok Q.I.M. (1983), « Dictionnaire et dérivation », Lexique, $\mathrm{n}^{\circ}$ 2, Lille : PUL, p. 69-77.

Molho M. (1982), «Grammaire analogique, grammaire du sens », Langages, $n^{\circ} 82$, Paris : Larousse, p. 41-51.

Nagórko A. (1998), Zarys gramatyki polskiej ze stowotwórstwem, Warszawa : PWN.

Nyrop K. (1913), Grammaire historique de la langue française, vol. IV, Copenhague : Ed. Gyldendalske Boghandel Nordisk Forlag.

Polański K. (réd.) (1999), Encyklopedia językoznawstwa ogólnego, Wroclaw : Ossolineum.

Ruwet N. (1972), Théorie syntaxique et syntaxe du français, Paris : Seuil.

Sękowska E. (1999), « Budowa wyrazów », in : Nauka o języku dla polonistów, dir. S. Dubisz, Warszawa : KiW.

Song J.J. (1996), Causatives and Causation: A Universal-Typological Perspective, London-New York : Longman.

Temple M. (1996), Pour une sémantique des mots construits, Villeneuve d'Ascq. : Presses universitaires de Septentrion.

Trésor de la langue française informatisé, http://atilf.atilf.fr/tll.htm 